\title{
Atomically-Resolved Characterization of
}

\section{Optically-Driven Ligand Reconfiguration on}

\section{Nanoparticle Catalyst Surfaces}

Mary O. Olagunju, 1,* Yang Liu, 2,; Anatoly I. Frenkel, ${ }^{2,3, *}$ Marc R. Knecht, 1,4,*

1. Department of Chemistry, University of Miami, 1301 Memorial Drive, Coral Gables, Florida

33146, United States

2. Department of Materials Science and Chemical Engineering, Stony Brook University, Stony

Brook, New York 11794, USA.

3. Chemistry Division, Brookhaven National Laboratory, Upton, New York 11973, USA.

4. Dr. J.T. Macdonald Foundation Biomedical Nanotechnology Institute, university of Miami,

UM Life Science Technology Building, 1951 NW $7^{\text {th }}$ Ave, Suite 475, Miami, Florida 33136,

USA. 


\section{SUPPORTING INFORMATION}

*To whom correspondence should be address: MRK- $\underline{\text { knecht@miami.edu; }}$ AIF = anatoly.frenkel@stonybrook.edu 
SUPPORTING INFORMATION
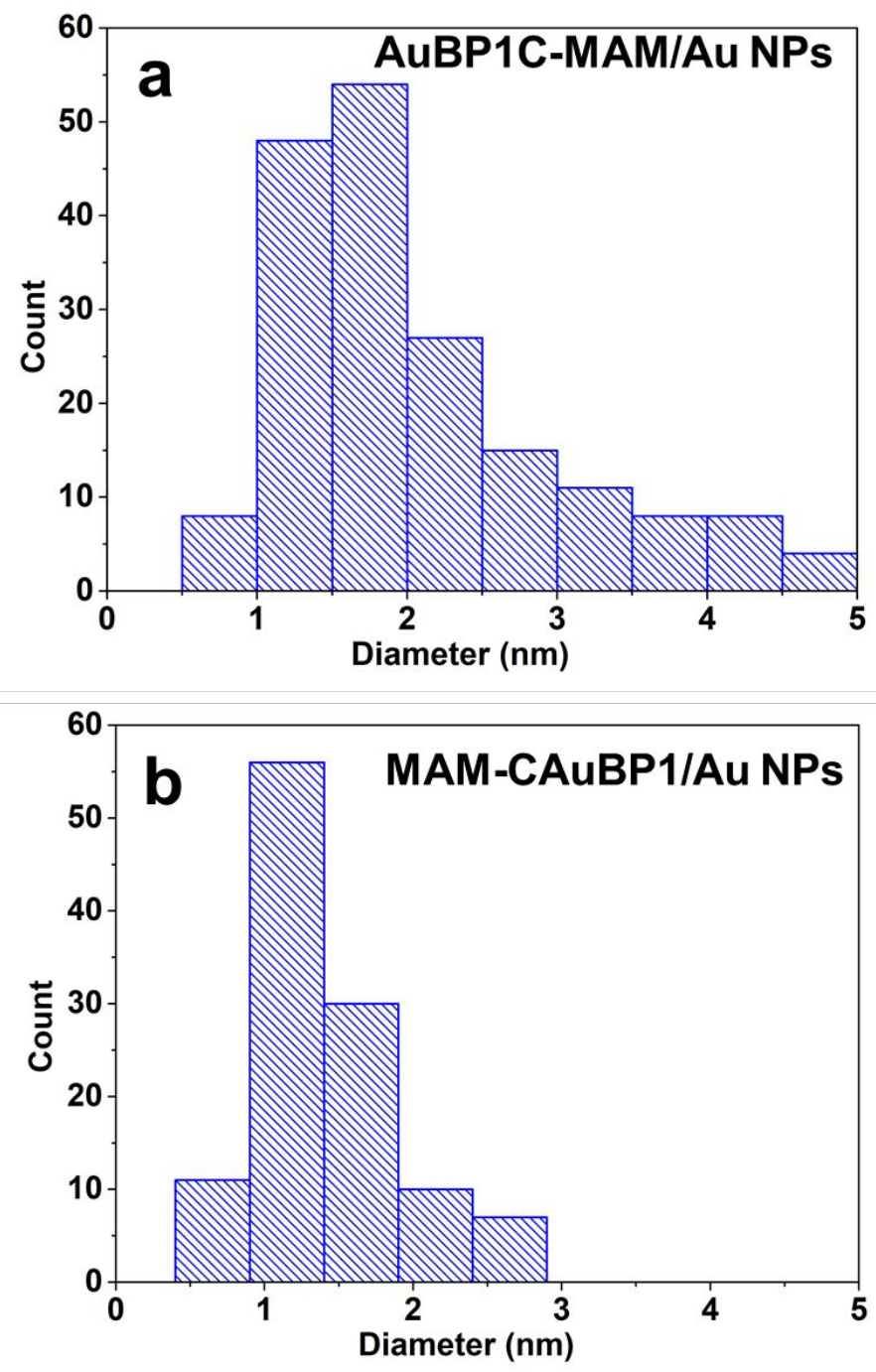

Figure S1. Particle sizing histograms for the Au NPs capped with (a) AuBP1C-MAM and (b) MAMCAuBP1. 


\section{SUPPORTING INFORMATION}
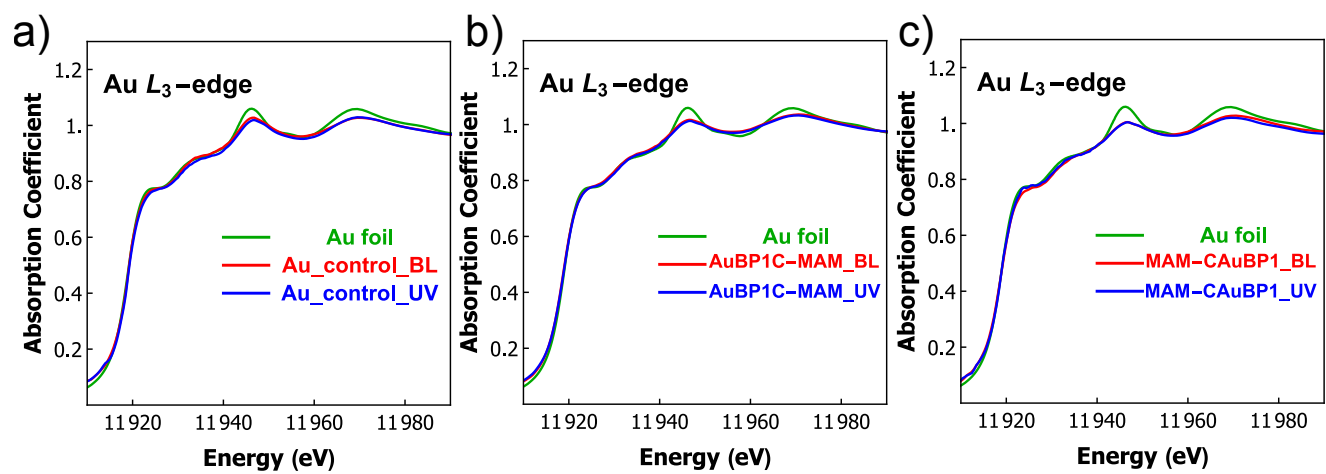

Figure S2. XANES spectra at $\mathrm{Au} \mathrm{L}_{3}$-edge of $\mathrm{Au}$ foil and (a) Au control, (b) AuBP1C-MAM-capped $\mathrm{Au}$ NPs, and (c) MAM-CAuBP1-capped Au NPs. BL represents before UV light exposure (i.e., trans), while UV represents after UV light exposure (i.e., cis). 


\section{SUPPORTING INFORMATION}
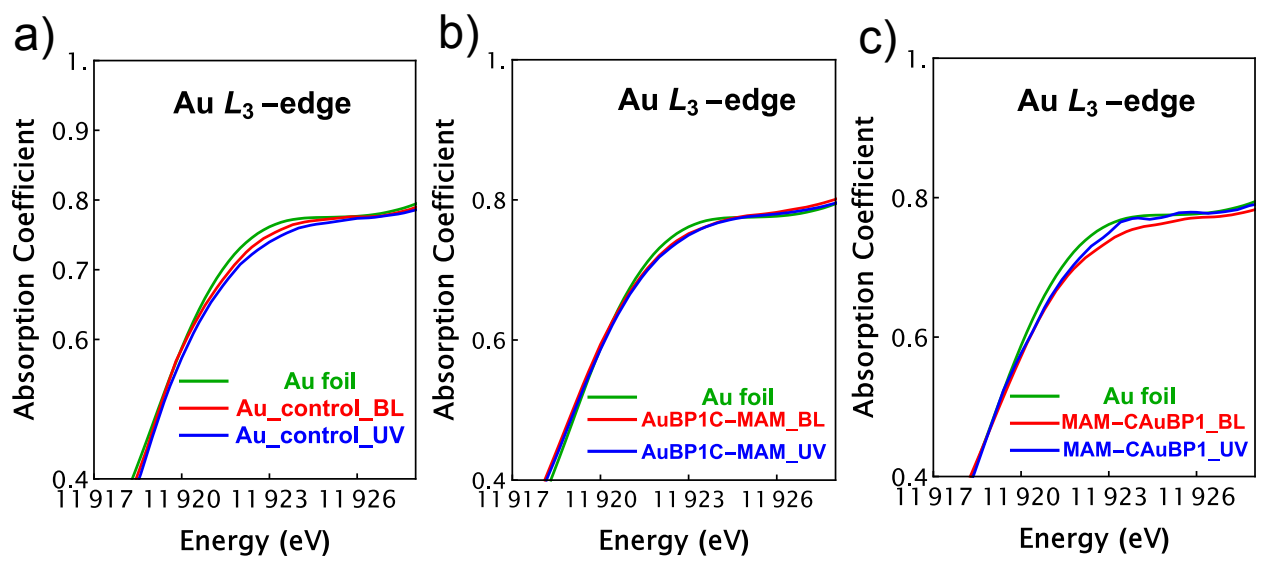

Figure S3. XANES spectra at $\mathrm{Au} \mathrm{L}_{3}$-edge of $\mathrm{Au}$ foil and for (a) Au control, (b) AuBP1C-MAM-capped Au NPs, and (c) MAM-CAuBP1-capped Au NPs. BL represents before UV light exposure (i.e., trans), while UV represents after UV light exposure (i.e., cis). 


\section{SUPPORTING INFORMATION}

\begin{tabular}{|c|c|c|c|c|}
\hline Sample & k-weight & k-range $\left(\AA^{-1}\right)$ & $\operatorname{dk}\left(\AA^{-1}\right)$ & R-range (Å) \\
\hline $\mathrm{Au}$ _Control_BL & 2 & $2-8$ & 1 & $1.3-3.44$ \\
\hline $\mathrm{Au} \_$Control_UV & 2 & $2-8$ & 1 & $1.3-3.44$ \\
\hline $\begin{array}{l}\text { AuBP1C- } \\
\text { MAM_BL }\end{array}$ & 2 & $2-8$ & 1 & $1.2-3.44$ \\
\hline $\begin{array}{l}\text { AuBP1C- } \\
\text { MAM UV }\end{array}$ & 2 & $2-8$ & 1 & $1.2-3.44$ \\
\hline $\begin{array}{c}\text { MAM- } \\
\text { CAuBP1_BL }\end{array}$ & 2 & $2-8$ & 1 & $1.3-3.44$ \\
\hline $\begin{array}{c}\text { MAM- } \\
\text { CAuBP1 UV }\end{array}$ & 2 & $2-8$ & 1 & $1.3-3.44$ \\
\hline
\end{tabular}

Table S1. Fourier transform parameters. BL represents before UV light exposure (i.e., trans), while UV represents after UV light exposure (i.e., cis). 


\section{SUPPORTING INFORMATION}

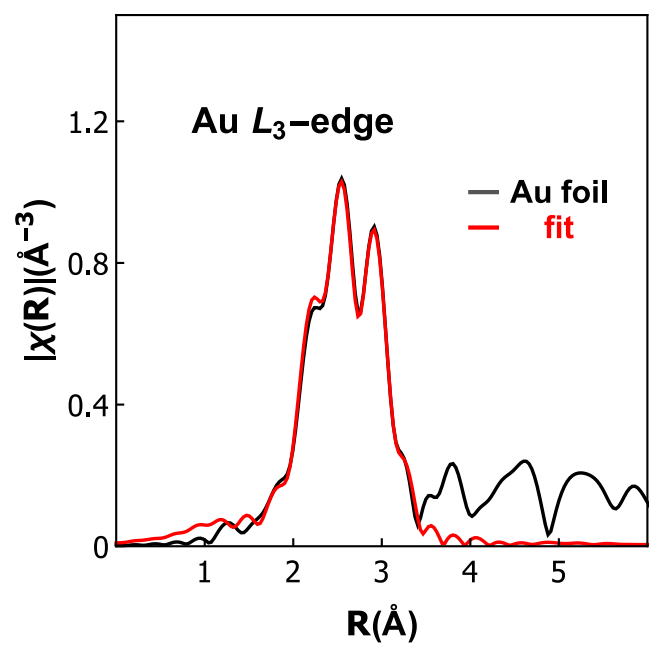

Figure S4. The fitting results for the Au foil 


\section{SUPPORTING INFORMATION}
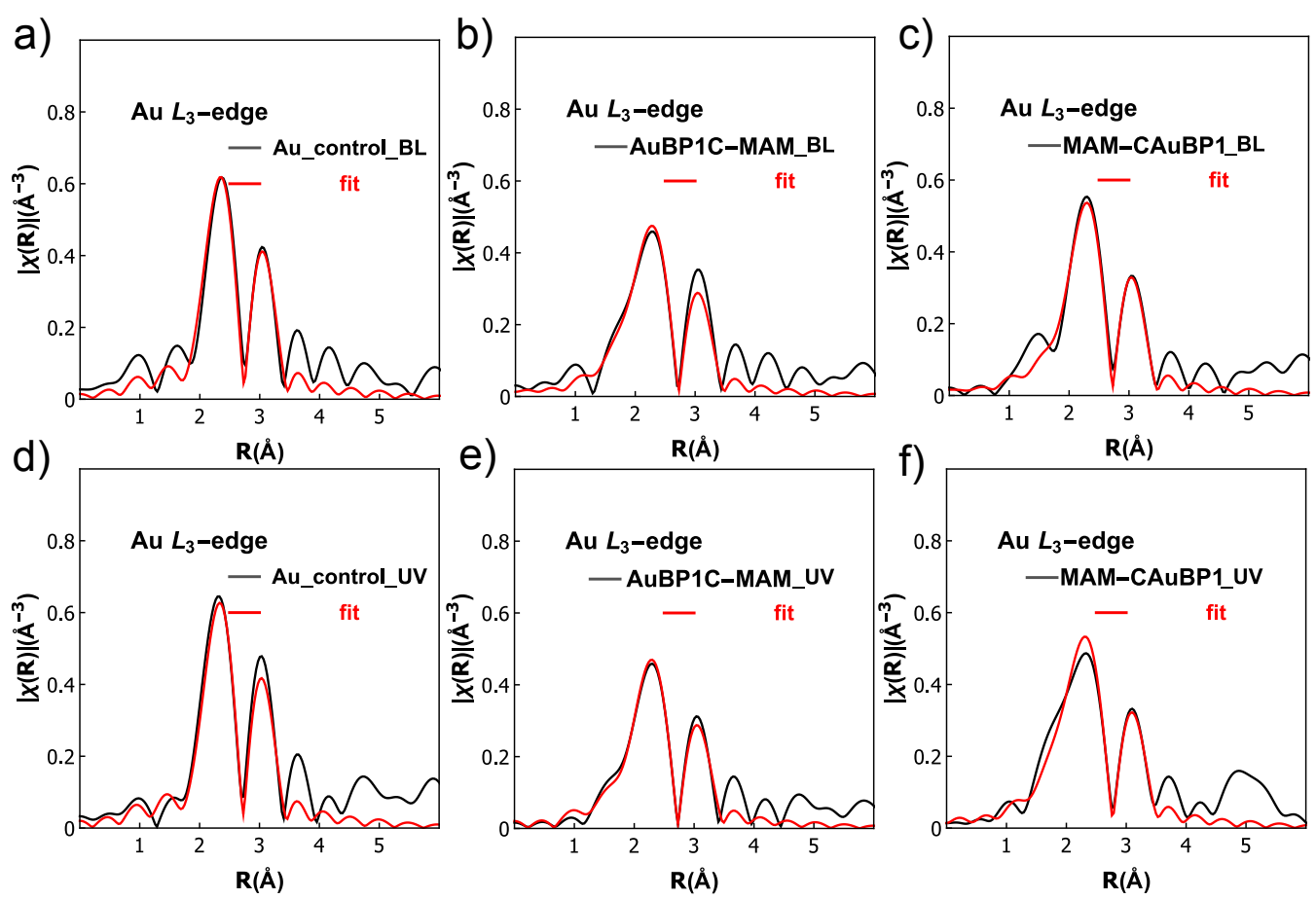

Figure S5. The fitting results for the (a) Au control_BL, (b) AuBP1C-MAM_BL, (c) MAM-CAuBP1_BL, (d) Au control_UV, (e) AuBP1C-MAM_UV and (f) MAM-AuBP1C_UV. BL represents before UV light exposure (i.e., trans), while UV represents after UV light exposure (i.e., cis). 